\title{
Antioxidant and anti-excitotoxicity effect of Gualou Guizhi decoction on cerebral ischemia/reperfusion injury in rats
}

\author{
SHENGNSAN ZHANG ${ }^{1}$, YUQIN ZHANG ${ }^{1}$, HUANG LI $^{1}$, WEI XU ${ }^{1}$, \\ $\mathrm{KEDAN} \mathrm{CHU}^{1}$, LIDIAN CHEN ${ }^{2}$ and XIANWEN CHEN ${ }^{1}$ \\ Colleges of ${ }^{1}$ Pharmacy and ${ }^{2}$ Rehabilitative Medicine, Fujian University of Traditional Chinese Medicine, \\ Fuzhou, Fujian 350122, P.R. China
}

Received March 30, 2014; Accepted November 4, 2014

DOI: $10.3892 /$ etm.2015.2386

\begin{abstract}
Stroke is the leading cause of disability in adults and the second most common cause of mortality worldwide. There is currently intense interest in the use of natural products in the treatment of the condition. The aim of this study was to investigate the effect of Gualou Guizhi decoction (GLGZD) on rats subjected to cerebral ischemia/reperfusion injury and the possible mechanisms involved. Cerebral ischemia/reperfusion injury was induced by the middle cerebral artery occlusion method. Ischemic injury was assessed by estimating neurological function and measuring brain infarct volume, and the terminal deoxynucleotidyl transferase-mediated dUTP nick end labeling method was employed to examine ischemia-induced apoptosis. The levels of the antioxidative enzyme superoxide dismutase (SOD) and the concentrations of the non-enzymatic scavenger glutathione (GSH) and malondialdehyde (MDA) were measured to investigate the antioxidant mechanisms. In addition, the levels of excitatory amino acids (EAAs) and glutamate receptor 1 (GluR1) were examined using an automatic amino acid analyzer and immunohistochemical analysis. The administration of GLGZD attenuated the cerebral ischemia/reperfusion injury-induced neural deficits and cerebral infarct volume, reduced the levels of MDA and EAAs (glutamate and aspartate), significantly increased the activity of the antioxidant GSH and notably elevated the activity of SOD. Consistently, GLGZD inhibited ischemia-induced apoptosis and downregulated the expression of GluR1. In conclusion, this study suggested that GLGZD exerts a neuroprotective
\end{abstract}

Correspondence to: Professor Kedan Chu, College of Pharmacy, Fujian University of Traditional Chinese Medicine, 1 Huatuo Road, Fuzhou, Fujian 350122, P.R. China

E-mail: chukedan@gmail.com

Professor Lidian Chen, College of Rehabilitative Medicine, Fujian University of Traditional Chinese Medicine, 1 Huatuo Road, Fuzhou, Fujian 350122, P.R. China

E-mail: clidianlab@gmail.com

Key words: Gualou Guizhi decoction, focal cerebral ischemic/reperfusion injury, antioxidant, anti-excitotoxicity effect on focal cerebral ischemia/reperfusion injury through the modulation of multiple antioxidant and anti-excitotoxicity pathways.

\section{Introduction}

Stroke is a life-threatening disease that is considered the most common cause of disability in adults. According to the World Health Organization, 15 million individuals suffer from stroke worldwide each year. Of these, 5 million succumb and a further 5 million are permanently disabled (1). Ischemic stroke is by far the most common type of stroke, accounting for $\sim 88 \%$ of all stroke cases. Ischemic stroke results from a loss of blood supply to part of the brain, initiating the ischemic cascade (2).

The mechanisms involved in ischemic stroke have been suggested to involve a complex interplay of excitotoxicity, oxidative stress, inflammation and apoptosis (3-6); however, oxidative stress is considered to have a key role in the pathogenesis of ischemia/reperfusion injury (4). Reactive oxygen species are found to be over-produced during ischemia/reperfusion, accompanied by elevated levels of free radicals. It is known that reactive oxygen species are generated following $\mathrm{N}$-methyl-D-aspartate receptor activation (7), which enhances glutamate (Glu) release (8) and leads to excitotoxicity. This initiates a chain reaction or signaling pathways and leads to the process of cell death. Furthermore, it has been reported that oxidative stress and excitotoxicity may be interdependent mechanisms involved in neuronal cell injury and death (9).

To date, numerous antioxidants, such as vitamin $\mathrm{E}$ and edaravone have shown neuroprotective effects in ischemia/reperfusion-induced cerebral injury (10-12); however, these agents do not exhibit satisfactory clinical outcomes due to a variety of patient- and drug-associated factors. There has recently been increased interest in the application of natural products, particularly Traditional Chinese Medicine, for the treatment of stroke. Gualou Guizhi decoction (GLGZD), a well-known traditional Chinese formula, was first recorded in 'Essentials from the Golden Cabinet', which was written during the Eastern Han Dynasty, in 210 A.D (13). According to the theory of Traditional Chinese Medicine, GLGZD is formulated of six herbs, which collectively exert therapeutic and modulatory effects. The formula has been used to treat muscular spasticity following stroke, epilepsy or spinal cord 
injury in China (14-16). The present authors' clinical study revealed the promising effects of GLGZD in stroke patients (Zhang et al, unpublished data); however, although reports from a few clinical studies are available (17), little investigation has been performed into the mechanism underlying the action of GLGZD against cerebral ischemia/reperfusion injury. The present study was therefore designed to confirm the potential effects of GLGZD on focal cerebral ischemia/reperfusion injury and elucidate the underlying therapeutic mechanism by using a middle cerebral artery occlusion (MCAO) model of cerebral ischemia.

\section{Materials and methods}

Animals and materials. Male Sprague Dawley (specific pathogen-free) rats, weighing $300 \pm 20 \mathrm{~g}$, were provided by the Laboratory Animal Center of Fujian University of Traditional Chinese Medicine (Fuzhou, China). All experiments were conducted in accordance with the Institutional Animal Care and Use Committee of Fujian University of Traditional Chinese Medicine.

The six herbal medicines of GLGZD were purchased from Tongchun Drugstore (Fuzhou, China) and identified by Professor Chengzhi Yang (College of Pharmacy, Fujian University of Traditional Chinese Medicine). Acetonitrile was high-performance liquid chromatography grade and purchased from Merck Co. (Darmstadt, Germany). Deionized water used throughout the experiments was generated by a Millipore water purification system (Milli-Q ${ }^{\circledR}$ Direct-Q 3; Millipore, Milford, MA, USA). All other chemicals were obtained from commercial sources unless otherwise stated.

Preparation of GLGZD. GLGZD was prepared from the six herbs (Trichosanthes kirilowii Maxim., Paeonia lactiflora Pall., Cinnamomum cassia Presl., Glycyrrhiza uralensis Fisch., Zingiber officinale Rosc. and Ziziphus jujuba Mill) with the ratio of 10:3:3:3:2:3 (dry weight), respectively, and extracted with $80 \%$ ethanol twice, $1 \mathrm{~h}$ per time. Filtrate was recovered from the ethanol and concentrated to extraction with a relative density of $1.2\left(50^{\circ} \mathrm{C}\right)$. The decoction was obtained for further use.

Focal cerebral ischemia/reperfusion model. The focal cerebral ischemia/reperfusion model was generated using MCAO methodology, as described previously (18). Briefly, rats were anesthetized with chloral hydrate $(350 \mathrm{mg} / \mathrm{kg})$ intraperitoneally, and the common left carotid artery, external carotid artery (ECA) and internal carotid artery (ICA) were exposed. A 3-0 surgical monofilament nylon suture with a rounded tip was carefully inserted from the ECA into the ICA and was advanced to occlude the origin of the left MCA, until a light resistance was felt (18-22 $\mathrm{mm})$. After $2 \mathrm{~h}$ of occlusion, the nylon suture was withdrawn for blood reperfusion. During the surgical procedures, the body temperature of the rats was maintained at $37 \pm 0.5^{\circ} \mathrm{C}$. Following surgery, the rats were allowed to recover in pre-warmed cages. Sham-operated rats underwent the same procedure, but arteries were not occluded. Successfully established rat models of focal cerebral ischemia/reperfusion were divided into two experimental groups to give three groups ( $n=15$ per group) in total: Sham surgery, model and GLGZD.
Drug administration protocol. GLGZD was administered at doses of $7.2 \mathrm{~g} / \mathrm{kg}$ once a day, starting $2 \mathrm{~h}$ after reperfusion, between days 1 and 7 . Saline solution $(0.9 \%)$ was administered to the sham surgery and model groups.

Evaluation of neurological deficit score. Neurological deficit was scored using the criteria of a five-point scale (19), as follows: Score 0 , no neurological symptoms; score 1, inability to completely extend the front jaw on the contralateral side; score 2 , rotation while crawling and falling to the contralateral side; score 3 , inability to walk without assistance and score 4 , unconsciousness. Rat behavioral tests were performed after 60 min of ischemia followed by 7 days of exercise. The evaluation was blindly performed by a single observer.

Evaluation of cerebral infarct volume. Six rats were sacrificed by decapitation and the intact brain was obtained on the seventh day after MCAO in each group. The frozen brains were sliced into uniform coronal sections, and then immediately stained with 2\% 2,3,5-triphenyltetrazolium chloride (TTC) solution in phosphate buffer ( $\mathrm{pH}$ 7.4). The sections were kept at $37^{\circ} \mathrm{C}$ for $1 \mathrm{~h}$ and turned over several times. Following staining, the infarct volume was measured using image analyzer software (Motic Med 6.0; Xiamen Motic Software Engineering Co., Ltd., Shenzhen, China) and calculated as a percentage fraction of non-viable cerebral tissue of the global brain. Color images of these slices were captured using a digital camera (Canon 550D; Canon Inc., Tokyo, Japan).

Biochemical assays. The activity of superoxide dismutase (SOD), as well as the concentrations of glutathione (GSH) and malondialdehyde (MDA), in the blood samples were measured using total superoxide dismutase (T-SOD), cell MDA and reduced GSH assay kits from Nanjing Jiancheng Institute of Bioengineering (Nanjing, China) in accordance with the manufacturer's instructions.

Determination of EAA level in cerebrospinal fluid. Cerebrospinal fluid was collected for the determination of Glu and aspartate (Asp) levels by a Hitachi automatic L-8900 amino acid analyzer (Hitachi, Tokyo, Japan) (20).

Immunohistochemical analysis of Glu receptor 1 (GluRl). Six rats in each group were anesthetized and were fixed with a buffered $4 \%$ paraformaldehyde solution by transcardial perfusion. The intact brain was then embedded with paraffin, and paraffin-embedded sections were used for the GluR1 immunohistochemistry assay. In brief, paraffin sections were dewaxed and incubated in boiling citrate buffer for antigen retrieval. The sections were subsequently incubated with polyclonal rabbit anti-GluR1 primary antibodies (1:500; \#bs-10042R; Beijing Biosynthesis Biotechnology Co., Ltd., Beijing China) at room temperature for $2 \mathrm{~h}$, following incubation with $3 \% \mathrm{H}_{2} \mathrm{O}_{2}$ and blocking by normal goat serum. Following a rinse in phosphate-buffered saline (PBS), the sections were incubated with a biotinylated polyclonal anti-rabbit IgG/streptavidin secondary antibody (1:1,000; \#bs-0295G-SA; Beijing Biosynthesis Biotechnology Co., Ltd.), washed and incubated with horseradish peroxidase-labeled streptavidin (Beijing Biosynthesis Biotechnology Co., Ltd.). The sections were then 


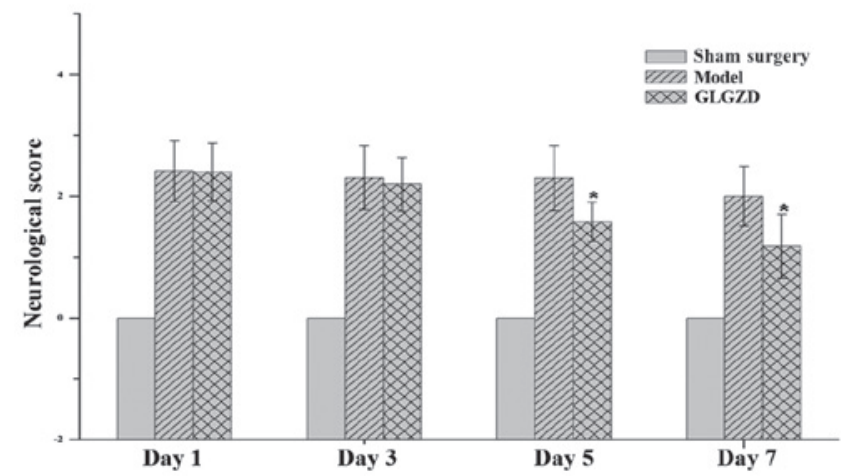

Figure 1. Effect of GLGZD on the development of behavioral abnormalities following MCAO. Neurological score was taken as the index. Data are presented as the mean \pm standard deviation in each group. ${ }^{*} \mathrm{P}<0.05$, vs. the MCAO model group. GLGZD, Gualou Guizhi decoction; MCAO, middle cerebral artery occlusion.
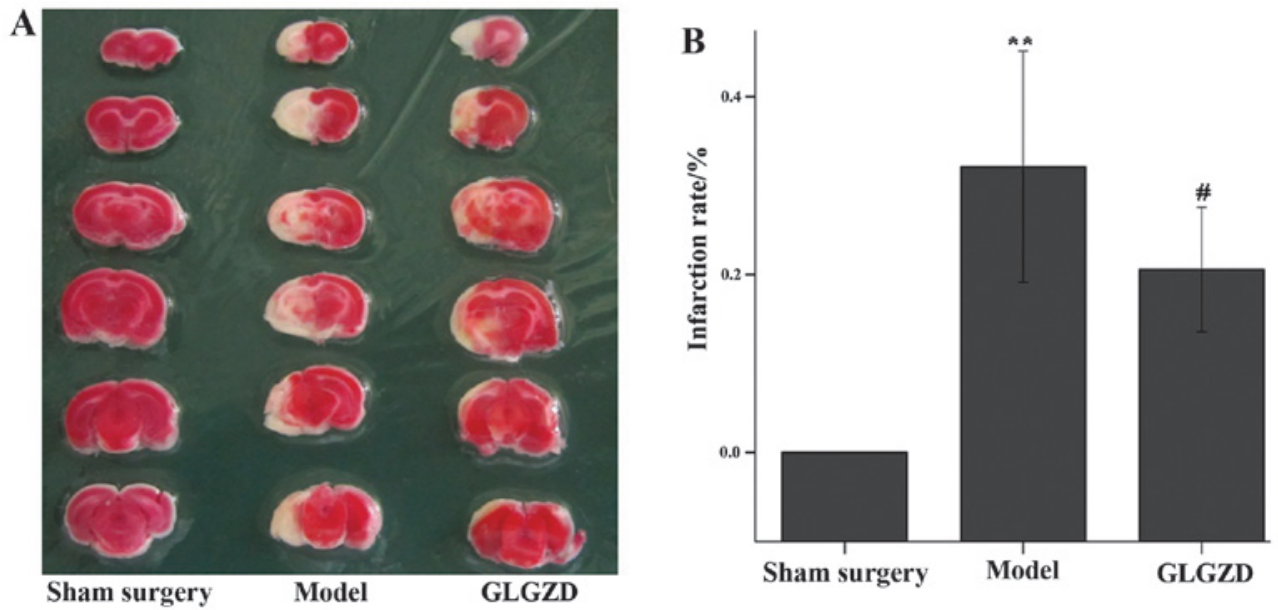

Figure 2. Effect of GLGZD on cerebral infarction in MCAO model rats. (A) Representative 2,3,5-triphenyltetrazolium chloride-stained brain coronal sections of four groups. The images were taken by a high-resolution digital camera. The normal tissue was stained red-blue and the infarct area was stained pale pink. (B) Infarct volume was expressed by bar graphs and quantified using the Motic Med 6.0 system. ${ }^{* *} \mathrm{P}<0.01$, vs. the sham surgery group, ${ }^{\#} \mathrm{P}<0.05$, vs. the model group. GLGZD, Gualou Guizhi decoction; MCAO, middle cerebral artery occlusion.

developed with diaminobenzidine and counterstained with hematoxylin. PBS replaced the primary antibody to determine specific binding for the negative control. Microscopic images were acquired using a Leica microscope (DM4000B; Leica Microsystems GmbH, Wetzlar, Germany) and five high-power fields (magnification, x400) were randomly selected in each slide. The average proportion of positive cells in each field was calculated by the true color multi-functional cell image analysis management system (Image-Pro Plus; Media Cybernetics, Inc.).

Terminal deoxynucleotidyl transferase-mediated dUTP nick end labeling (TUNEL) staining. TUNEL staining was performed in accordance with the manufacturer's instructions using an apoptosis detection kit (Promega Corp., Madison, WI, USA). The apoptotic index was calculated by multiplying the quantity and staining intensity scores (21).

Statistical analysis. Data are presented as the mean \pm standard deviation. The statistical significance of differences was analyzed by one-way analysis of variance. $\mathrm{P}<0.05$ was considered to indicate a statistically significant difference.

\section{Results}

Effect of GLGZD on neurological deficits and cerebral infract volume. According to the criteria of the five-point scale, neurological symptoms were not observed in the sham surgery group, but were evident in the two cerebral ischemia/reperfusion groups (Fig. 1). GLGZD treatment for the first 3 days did not significantly reduce the neurological deficit scores compared with the model group; thereafter, however, the scores were significantly reduced by GLGZD $(\mathrm{P}<0.01)$.

Following TTC staining, the living cells were stained deep red, while the infarcted cells remained pale due to dehydrogenase loss at 7 days after ischemia (Fig. 2A). GLGZD significantly decreased the infarct volume to $0.20 \pm 0.06$ $(\mathrm{P}<0.05)$, as compared with the cerebral ischemia/reperfusion group (0.32 \pm 0.05$)$ (Fig. 2B).

Effect of GLGZD on SOD activity and MDA and GSH levels in the blood. As shown in Table I, the levels of MDA and GSH were $2.120 \pm 0.79 \mathrm{nmol} / \mathrm{ml}$ and $18.231 \pm 0.90 \mu \mathrm{mol} / \mathrm{ml}$, respectively, and the SOD activity was $20.213 \pm 0.02 \mathrm{U} / \mathrm{ml}$ 
Table I. Effect of GLGZD on SOD activity and MDA and GSH levels in the blood of rats subjected to cerebral ischemia/reperfusion injury.

\begin{tabular}{lccc}
\hline Groups & SOD $(\mathrm{U} / \mathrm{ml})$ & MDA $(\mathrm{nmol} / \mathrm{ml})$ & GSH $(\mu \mathrm{mol} / \mathrm{l})$ \\
\hline Sham surgery & $20.213 \pm 0.02$ & $2.120 \pm 0.79$ & $18.231 \pm 0.90$ \\
Model & $3.689 \pm 0.03^{\mathrm{a}}$ & $5.680 \pm 1.25^{\mathrm{a}}$ & $3.001 \pm 0.29^{\mathrm{a}}$ \\
GLGZD & $19.686 \pm 0.02^{\mathrm{b}}$ & $2.190 \pm 0.29^{\mathrm{b}}$ & $16.539 \pm 1.30^{\mathrm{b}}$ \\
\hline
\end{tabular}

Values are presented as the mean \pm standard deviation. ${ }^{\mathrm{a}} \mathrm{P}<0.01 \mathrm{vs}$. the sham surgery group; ${ }^{\mathrm{b}} \mathrm{P}<0.01 \mathrm{vs}$. the MACO model group. GLGZD, Gualou Guizhi decoction; MCAO, middle cerebral artery occlusion; SOD, superoxide dismutase; MDA, malondialdehyde; GSH, glutathione.

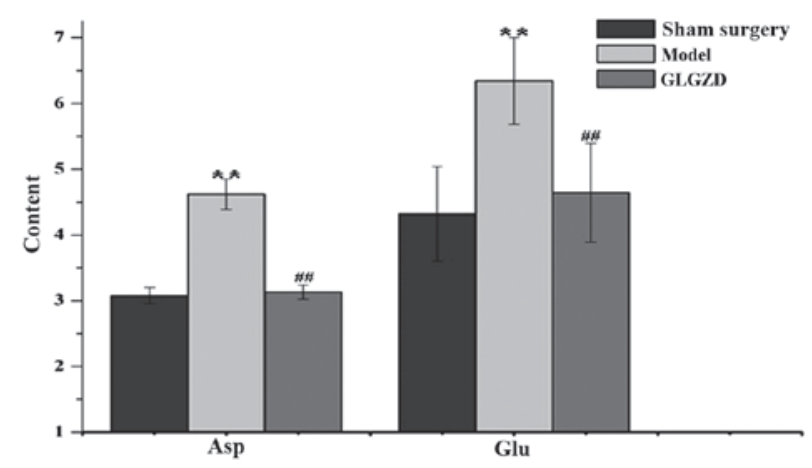

Figure 3. Effect of GLGZD on excitatory amino acid (Glu and Asp) levels in cerebrospinal fluid. ${ }^{* *} \mathrm{P}<0.05$, vs. the sham surgery group, ${ }^{\# \#} \mathrm{P}<0.01$, vs. the MCAO model group. GLGZD, Gualou Guizhi decoction; MCAO, middle cerebral artery occlusion; Glu, glutamate; Asp, aspartate.
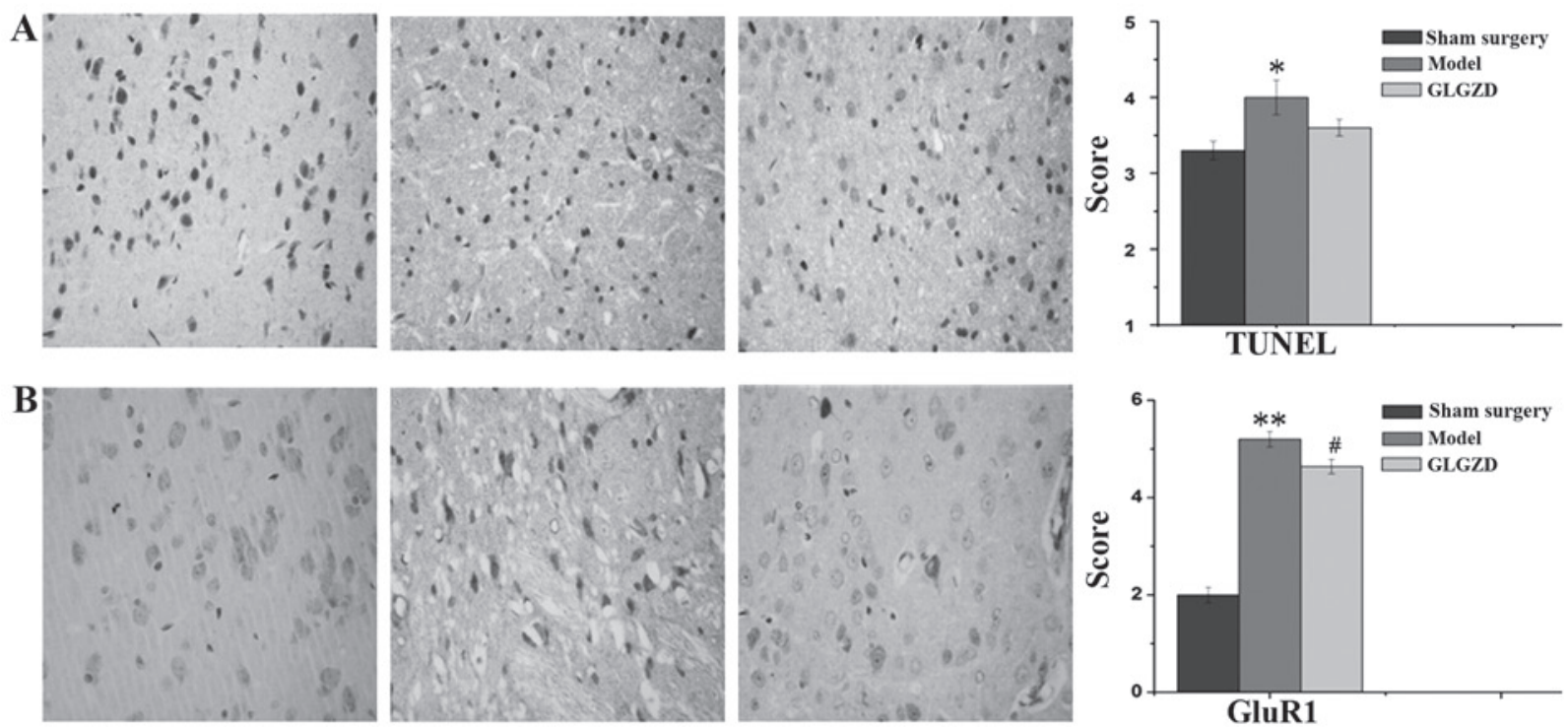

Figure 4. Effects of GLGZD treatment on (A) apoptosis and (B) the expression of GluR1 in MCAO-induced ischemic rat brain tissues, as assessed by TUNEL staining and immunohistochemical analysis, respectively (original magnification, $\mathrm{x} 400$ ). ${ }^{*} \mathrm{P}<0.05$ and ${ }^{* *} \mathrm{P}<0.01$ vs. the sham surgery group, ${ }^{*} \mathrm{P}<0.05$, vs. the MCAO model group. GLGZD, Gualou Guizhi decoction; MCAO, middle cerebral artery occlusion; GluR1, glutamate receptor 1; TUNEL, terminal deoxynucleotidyl transferase-mediated dUTP nick end labeling.

in the sham surgery group; however, the SOD activity and GSH levels were markedly decreased and the MDA level was significantly increased in the model group compared with those in the sham surgery group. In the GLGZD group, it was observed that the MDA and GSH levels and SOD activity were significantly altered when compared with those in the model group $(\mathrm{P}<0.05)$.
Effect of GLGZD on EAAs in cerebrospinal fluid. In the model group, the levels of EAAs, such as Glu and Asp, were increased by 150 and $146 \%$, respectively $(\mathrm{P}<0.05)$, compared with those in the sham surgery group. Following treatment with GLGZD, the EAA levels were decreased by 36 and $47 \%$, respectively, compared with those in the model group $(\mathrm{P}<0.05)$ (Fig. 3). 
Effect of GLGZD on apoptosis in the brain. Apoptosis (programmed cell death) was detected by TUNEL assay. Data in Fig. 4A show that GLGZD treatment decreased the proportion of TUNEL-positive cells compared with the model group, thus showing the anti-apoptotic activity of GLGZD in vivo.

Effect of GLGZD on the expression of GluRl in brain. The protein expression of GluR1 was evaluated via immunohistochemical analysis. Protein expression was significantly elevated in the model group compared with that in the sham surgery group, while decreased expression was observed in the GLGZD group compared with that in the model group (Fig. 4B).

\section{Discussion}

Ischemic stroke is the most commonly encountered type of stroke in humans. In experimental stroke, the two models used are the permanent MCAO model and the focal cerebral ischemia model. The focal cerebral ischemia model more closely replicates the condition in human beings; therefore, a reversible model of focal ischemia (i.e. the temporary MCAO model) is more relevant than the permanent occlusion model (22). The major advantages of this model are that the method is relatively simple and that the MCA can be occluded and reperfused without craniotomy $(23,24)$.

In the present study, the neuroprotective effect of GLGZD was evaluated in vivo using an MCAO rat model. The results showed that GLGZD significantly decreased the focal infarct volume, neurological deficit score and level of apoptosis compared with the model rats. These findings were consistent with those of a previous report (18).

The neuroprotective effect of GLGZD may involve a number of different mechanisms, including reduced inflammatory cytokine levels (25), an antioxidation effect (26) and the modulation of Glu levels and $\alpha$-amino-3-hydroxy-5-methyl-4-isoxazolepropionic acid receptor (AMPAR) expression (18). In the MCAO rats, a significant increase was observed in the level of MDA and a decrease in the levels of endogenous antioxidants, i.e. SOD and GSH. This was indicative of marked oxidative stress. Excessive free radical generation occurs at the time of reperfusion and accounts for free radical-induced injury (27). In the GLGZD-treated rats, a significant attenuation in the level of MDA and increases in the activity of SOD and level of GSH were noted.

Oxidative stress can cause cellular damage and enhance Glu release, thus leading to excitotoxicity. Glu is the most common excitatory neurotransmitter in the central nervous system and is involved in numerous aspects of normal brain function; however, abnormally elevated levels of Glu may induce excitatory neural toxicity (28). Excess Glu-induced excitotoxicity is involved in the pathogenesis of several human neurological diseases, such as stroke. It has been reported that cerebral ischemia or brain injury markedly elevates Glu concentrations (29), with the resulting excitotoxicity leading to neuronal injury or death (30). The present study showed that Glu and Asp concentrations were significantly decreased in GLGZD-treated rats compared with those in the model rats; similar results were observed for GluR1 expression. GluR1 is one of the AMPAR subunits. AMPAR is a member of the ionotropic glutamate receptor family and mediates excitatory synaptic transmission in the brain. It additionally has an important role in motor function following cerebral ischemia. Gottlieb and Matute (31) found that AMPAR subunits were upregulated in immunoreactive hypertrophic astrocytes in the CA1 hippocampal region following transient forebrain ischemia (31); therefore, a normal Glu concentration is important to prevent glutamate-induced neurotoxicity.

In this study, initial evidence was provided that GLGZD exerts a neuroprotective effect in vivo by promoting endogenous antioxidant enzymatic activities, decreasing neuronal cell death and Glu concentration and inhibiting GluR1 expression. The findings lead to the speculation that GLGZD may be a potential therapeutic agent for cerebral ischemia.

\section{Acknowledgements}

This study was carried out in the State Key Laboratory of Chinese Pharmacies of the Fujian Provincial Department of Science and Technology, the Collaborative Innovation Center for Rehabilitation Technology and the TCM Rehabilitation Research Center of SATCM. It was funded by the Important Subject of Fujian Province Science and Technology Hall of China (no. 2012Y0041) and the Important Subject of Fujian Province Education Hall of China (no. JA12176).

\section{References}

1. World Health Organization: The World Health Report 2002. World Health Organization, France, 2002.

2. Deb P, Sharma S and Hassan KM: Pathophysiologic mechanisms of acute ischemic stroke: An overview with emphasis on therapeutic significance beyond thrombolysis. Pathophysiology 17: 197-218, 2010.

3. Candelario-Jalil E Injury and repair mechanisms in ischemic stroke: considerations for the development of novel neurotherapeutics. Curr Opin Investig Drugs 10: 644-654, 2009.

4. Janardhan V and Qureshi AI: Mechanisms of ischemic brain injury. Curr Cardiol Rep 6: 117-123, 2004.

5. Amantea D, Nappi G, Bernardi G, et al: Post-ischemic brain damage: pathophysiology and role of inflammatory mediators. FEBS J 276: 13-26, 2009.

6. Doyle KP, Simon RP and Stenzel-Poore MP: Mechanisms of ischemic brain damage. Neuropharmacology 55: 310-318, 2008.

7. Lafon-Cazal M, Pietri S, Culcasi M, et al: NMDA-dependent superoxide production and neurotoxicity. Nature 364: 535-537, 1993.

8. Gilman SC, Bonner MJ and Pellmar TC: Peroxide effects on $[3 \mathrm{H}] \mathrm{L}$-glutamate release by synaptosomes isolated from the cerebral cortex. Neurosci Lett 140: 157-160, 1992.

9. Won MH, Kang T, Park S, et al: The alterations of N-Methyl-D-aspartate receptor expressions and oxidative DNA damage in the CA1 area at the early time after ischemia-reperfusion insult. Neurosci Lett 301: 139-142, 2001.

10. El-Abhar HS: Possible neuroprotective effects of melatonin against ischemia/reperfusion insult in rat brain. Med Sci Res 27: 605-608, 1999.

11. Bora KS and Sharma A: Neuroprotective effect of Artemisia absinthium L. on focal ischemia and reperfusion-induced cerebral injury. J Ethnopharmacol 129: 403-409, 2010.

12. Yoneda Y, Uehara T, Yamasaki H, et al: Hospital-based study of the care and cost of acute ischemic stroke in Japan. Stroke 34: 718-724, 2003.

13. Zhang Z, Lin Y, Yang P, Hou X and Yang Y (eds): Synopsis of Golden Chamber. Macmillan Press, Beijing, pp203-204, 2008.

14. Yang C, Chen L and Tao J: New usage of a classical formula-Gua Lou Gui Zhi decotion. Liaoning Zhong Yi Za Zhi. 8: 166-167, 2010. 
15. Sun X: Research on formula treating paralysis and spasticity from 'treatise on febrile and miscellaneous diseases'. Zhongguo Zhong Yi Ji Chu Yi Xue Za Zhi 8: 644-645, 2010 (In Chinese).

16. Zhang L and Ai H: Effects of Gua Lou Gui Zhi decoction on c-fos and c-jun in epileptic rats. Shi Yong Zhong Yi Yao Za Zhi 23: 21-22, 2005 (In Chinese).

17. Yang $\mathrm{C}$, Chen L and Tao J: New usage of a classical formula-Gua Lou Gui Zhi decoction. Liaoning Zhong Yi Za Zhi 8: 166-167, 2010 (In Chinese).

18. Huang J, Tao J, Xue X, et al: Gua Lou Gui Zhi decoction exerts neuroprotective effects on post-stroke spasticity via the modulation of glutamate levels and AMPA receptor expression. Int J Mol Med 31: 841-848, 2013.

19. Camerlingo M, Salvi P, Belloni G, et al: Intravenous heparin started within the first $3 \mathrm{~h}$ after onset of symptoms as a treatment for acute nonlacunar hemispheric cerebral infarctions. Stroke 36: 2415-2420, 2005.

20. Guo C, Tong L, Xi M, et al: Neuroprotective effect of calycosin on cerebral ischemia and reperfusion injury in rats. J Ethnopharmacol 144: 768-774, 2012.

21. Zhang Y, Xu W, Li H, et al: Therapeutic effects of total alkaloids of Tripterygium wilfordii Hook f. on collagen-induced arthritis in rats. J Ethnopharmacol 145: 699-705, 2013.

22. Hossmann KA: Cerebral ischemia: models, methods and outcomes. Neuropharmacology 55: 257-270, 2008.

23. Belayev L, Alonso OF, Busto R, et al: Middle cerebral artery occlusion in the rat by intraluminal suture. Neurological and pathological evaluation of an improved model. Stroke 27: 1616-1622, 1996.
24. Yu XY, Lin SG, Zhou ZW, et al: Tanshinone IIB, a primary active constituent from Salvia miltiorrhiza, exhibits neuro-protective activity in experimentally stroked rats. Neurosci Lett 417: 261-265, 2007.

25. Hu H, Li Z, Zhu X, et al: Gua Lou Gui Zhi decoction suppresses LPS-induced activation of the TLR4/NF- $\kappa \mathrm{B}$ pathway in BV-2 murine microglial cells. Int J Mol Med 31: 1327-1332, 2013.

26. Mao JJ, Li ZF and Guang J: Impacts of Gualou Guizhi decoction extract on the expression of NrF2 and HO-1 mRNA in oxidative stress PC12 cells. Shijie Zhongxiyi Jiehe Zazhi 8: 563-566, 2013 (In Chinese).

27. Yu XQ, Xue CC, Zhou ZW, et al: In vitro and in vivo neuroprotective effect and mechanisms of glabridin, a major active isoflavan from Glycyrrhiza glabra (licorice). Life Sci 82: 68-78, 2008.

28. Danbolt NC: Glutamate uptake. Prog Neurobiol 65: 1-105, 2001.

29. Parelkar NK and Wang JQ: Upregulation of metabotropic glutamate receptor8 mRNA expression in the rat forebrain after repeated amphetamine administration. Neurosci Lett 433: 250-254, 2008.

30. Bonde C, Noraberg J, Noer $\mathrm{H}$ and Zimmer J: Ionotropic glutamate receptors and glutamate transporters are involved in necrotic neuronal cell death induced by oxygen-glucose deprivation of hippocampal slice cultures. Neuroscience 136: 779-794, 2005

31. Gottlieb M and Matute C: Expression of ionotropic glutamate receptor subunits in glial cells of the hippocampal CA1 area following transient forebrain ischemia. J Cereb Blood Flow Metab 17: 290-300, 1997. 